\title{
Control político del servicio civil \\ El caso del gobierno de Puerto Rico
}

\author{
Political Control over the Civil Service: \\ The Case of the Government of Puerto Rico \\ Elizabeth Pérez-Chiqués y Edgar O. Bustos*
}

Resumen: Luego de contar con un sistema de servicio civil de carrera consolidado, el gobierno de Puerto Rico ha revertido a un sistema de administración de personal donde predominan los intereses político-partidistas, esto mediante la manipulación sistemática de las estructuras formales del servicio civil. Este estudio emplea una estrategia cualitativa de métodos mixtos con el fin de identificar las estructuras y prácticas informales que sostienen al patronazgo y facilitan la discriminación política, así como entender la experiencia y perspectiva de los empleados públicos de carrera dentro de un sistema incongruente, que promulga ser de mérito, pero que los somete a una administración de personal regida informalmente por criterios político-partidistas. El caso de Puerto Rico nos ayuda a entender cómo un sistema de mérito puede verse sometido por el poder de los partidos políticos mediante la institucionalización de prácticas neopatrimonialistas con el objeto de legitimar valores vinculados al patronazgo, manteniendo al servicio civil principalmente como un instrumento de coerción con fines políticos. Este caso reafirma que los remedios legales y las penalidades no son suficientes para mantener un sistema de mérito o para disminuir las prácticas de favoritismo y discriminación que conforman al patronazgo.

Palabras clave: servicio civil, patronazgo, mérito, discriminación política, politización.

Abstract: After having a consolidated career civil service system, the government of Puerto Rico has informally reverted to a personnel management system where political-partisan interests predominate through the use and systematic manipulation of the formal structures

*Elizabeth Pérez-Chiqués es profesora-investigadora en la División de Administración Pública del Centro de Investigación y Docencia Económicas (CIDE), Circuito Tecnopolo Norte 117, Fracc. Tecnopolo Pocitos II, Aguascalientes, Ags., 20313. Tel: (449)9945150 . Correo-e: elizabeth.perez@cide.edu. orciD: https://orcid.org/0000-0003-4432-718X. Edgar O. Bustos es profesor-investigador visitante del Centro de Investigación y Docencia Económicas (CIDE), División de Administración Pública, Carretera México-Toluca 3655, Lomas de Santa Fe, 01210, Ciudad de México, México. Tel: +52 555727 9800. Correo-e: edgar. bustos@cide.edu. ORCID: https://orcid.org/0000-0003-3902-8652.

Artículo recibido el 7 de octubre de 2020 y aceptado para su publicación el 13 de abril de 2021 . 
of the civil service. This study employs a qualitative mixed methods strategy in order to identify the informal structures that operate within government agencies, their instruments, and practices. It also seeks to understand the career of public employees within an incongruous system, which promulgates to be merit-based, but which subjects them to personnel management that is informally dominated by political-partisan criteria. The case of Puerto Rico helps us understand how a merit system can be subdued by the power of political parties through the institutionalization of neo-patrimonial practices that legitimize values linked to patronage, maintaining the civil service mainly as an instrument of coercion for political purposes. This case study reaffirms that legal remedies and penalties are not sufficient to maintain a merit system or reduce the practices of favoritism and discrimination that constitute patronage.

Keywords: civil service, patronage, merit, political discrimination, politicization.

\section{INTRODUCCION}

Tos servicios civiles de carrera son sistemas de gestión de recursos humanos que se refieren a un cuerpo normativo que regula las relaciones laborales entre el Estado y un grupo de empleados contratados por méritos profesionales y no por nombramiento o designación política (Light, 2006). Estos sistemas han sido promovidos como herramientas prometedoras contra la corrupción (por ej., Grindle, 2012; Meyer-Sahling et al., 2018; Dahlström et al., 2012; Schuster, 2017), ya que se considera que generan un tipo de protección dentro del aparato gubernamental que altera los incentivos de los empleados gubernamentales, así como de los políticos, cambiando de manera fundamental las bases sobre las cuales se relacionan (por ej., Arellano Gault et al., 2018). Además, los servicios civiles pueden considerarse la piedra angular del Estado, ya que sostienen o debilitan todo el sistema de gobierno (Repucci, 2012).

Pero ¿qué ocurre cuando estos sistemas que pretenden combatir la corrupción son corrompidos? Este artículo tiene como finalidad analizar las estructuras y prácticas informales que sostienen al patronazgo, así como las (micro)dinámicas entre los designados políticos y servidores de carrera en un servicio civil, en donde la gestión de los primeros distorsiona los valores rectores del sistema. El trabajo argumenta que, mediante la institucionalización de prácticas neopatrimoniales, un sistema de mérito puede verse sometido por el poder de los partidos políticos. Para inducir sobre ello, el trabajo estudia el caso de Puerto Rico, cuyas características ayudan a entender los mecanismos mediante los cuales un sistema formal de gestión de recursos humanos se puede utilizar para servir a fines patrimoniales y contrarios al mérito, lo que resulta en una distorsión de los valores del servicio civil con serias repercusiones para la administración pública. 
Puerto Rico es un caso relevante de estudio para comprender los efectos de la corrupción del mérito en la gestión de recursos humanos. Este territorio estadounidense cuenta formalmente con un sistema de servicio civil que fue creado hace más de un siglo. El sistema está apoyado por una legislación sólida respaldada no solo por sus instituciones locales, sino por las instituciones de Estados Unidos, cuya experiencia con este tipo de sistema es la más relevante en el continente. Incluso, Puerto Rico llegó a ser reconocido como el único lugar en el Caribe con un servicio civil funcional, lo cual se le atribuía a la presencia sostenida de Estados Unidos (Kearney, 1986). Por otra parte, como en prácticamente toda la región latinoamericana y caribeña, Puerto Rico tiene un contexto con burocracias e instituciones débiles, lo que ha permitido que la dinámica bipartidista se haya apoderado del sistema mediante prácticas patrimoniales (Pérez-Chiqués y Rubin, 2021).

La legislación inicial de Puerto Rico para establecer un servicio civil se remonta al ańo 1907, seguida de leyes de personal aprobadas en los ańos 1931 (Ley 88 de la Comisión del Servicio Civil), 1947 (Ley 345 de la Agencia Central de Personal), 1975 (Ley 5 de Personal del Servicio Público), 2004 (Ley 184 para la Administración de los Recursos Humanos en el Servicio Público) y en 2017 (Ley 8 para la Administración y Transformación de los Recursos Humanos del Gobierno de Puerto Rico). Esta legislación respalda el cumplimiento del principio del mérito y la no discriminación basada en criterios político-partidistas; prohibición que también está plasmada en la Constitución del Estado Libre Asociado de Puerto Rico de 1952 (Caballero-Fuentes, 2012). En particular, la Ley de Personal del Servicio Público de 1975 establecía que el mérito "regirá todo el servicio público, de modo que sean los más aptos los que sirvan al gobierno... sin discrimen por razones de raza, color, sexo, nacimiento, edad, origen o condición social, ni ideas políticas o religiosas" (3 L.P.R.A. \$1311).

Puerto Rico, como territorio estadounidense, también se rige por las mismas leyes federales y decisiones de la Corte Suprema que limitan la discriminación política y el patronazgo. En particular, decisiones de la Corte Suprema, como Elrod v. Burns (1976) y Rutan v. Partido Republicano de Illinois (1990), resultaron en prohibiciones claras sobre la utilización de la afiliación política en la administración de empleados públicos. En Elrod v. Burns, el Tribunal determinó que despedir a empleados públicos por motivos políticos era inconstitucional; prohibición que no aplica a los cargos que requieren de la lealtad política para el desempeńo de sus funciones (ej., puestos de confianza). En el fallo Rutan v. Partido Republicano, esta prohibición se extendió a ascensos, traslados y decisiones sobre reclutamiento y contrataciones (Nigro et al., 2007). 
A pesar de la infraestructura legal e institucional que se ha desarrollado para prevenir la discriminación política y mantener al mérito como el principio que rige la gestión del personal público, la administración pública en Puerto Rico cuenta con un sistema de patronazgo informal robusto, en el cual el criterio político permea los procesos de recursos humanos de los empleados públicos de carrera (Cordero-Nieves et al., 2016) — prácticas ilegales e inconstitucionales a la luz de la legislación actual que rige en Puerto Rico-. ¿Cómo se conforma y cómo persiste un sistema informal de patronazgo dentro de un sistema de servicio civil basado en el mérito que está altamente regulado? ¿Cuáles son las prácticas y estructuras informales que sostienen al sistema?

Para identificar las estructuras y prácticas informales que sostienen al patronazgo dentro de un sistema formal de servicio civil y cómo estas corrompen al valor del mérito, este artículo basa su análisis en la noción de neopatrimonialismo para examinar la hibridación entre ambas prácticas (las formales e informales), y su impacto en la gestión de recursos humanos en la administración pública en Puerto Rico. Para ello, este estudio de caso emplea una estrategia cualitativa mixta que incluye 29 entrevistas en profundidad con empleados públicos y análisis de 50 casos judiciales de discriminación política. Siguiendo esta estrategia metodológica, el análisis busca identificar las estructuras informales que operan dentro de las agencias de gobierno, sus instrumentos y prácticas. El estudio busca, además, entender la experiencia de los empleados públicos de carrera dentro de un sistema incongruente, que promulga ser de mérito, pero que sujeta a estos a una administración regida por criterios neopatrimonialistas.

La literatura concerniente al estudio de los servicios civiles en países latinoamericanos se ha centrado en analizar la politización de la burocracia desde una perspectiva macro o de análisis institucional-estructural (ej., Chudnovsky y Cafarelli, 2008; Iacoviello et al., 2017; Klingner, 1996). Sin embargo, poco se ha dicho sobre las (micro)dinámicas entre individuos mediante las cuales los partidos políticos distorsionan las reglas formales para ejercer coerción política sobre los servidores pertenecientes al servicio civil. Este análisis nos ayuda a entender cómo un sistema de mérito puede verse sometido por el poder de los partidos políticos y sugiere que las prácticas y estructuras de poder informales deben entenderse y tomarse en cuenta antes de diseñar y adoptar una reforma del servicio civil, o bien para conservar un servicio civil establecido. Así, este estudio de caso reafirma que los remedios legales y las penalidades no son suficientes para implementar un sistema de mérito (ej., Schuster, 2017) o para disminuir las prácticas de favoritismo y discriminación que conforman al patronazgo. 
CUADRO 1. Evolución histórica en la gestión de recursos humanos en Puerto Rico

\begin{tabular}{|c|c|c|c|}
\hline Etapa & Valores dominantes & Sistema dominante & Presión de cambio \\
\hline $\begin{array}{l}\text { Adhesión } \\
\text { estadounidense } \\
(1898-1916)\end{array}$ & $\begin{array}{l}\text { Responsabilidad } \\
\text { política y representación }\end{array}$ & $\begin{array}{l}\text { Gobierno por parte de élites } \\
\text { norteamericanas }+ \\
\text { Ley de servicio civil }\end{array}$ & $\begin{array}{l}\text { Institucionalización } \\
\text { económica, política, } \\
\text { jurídica y militar }\end{array}$ \\
\hline $\begin{array}{l}\text { Acta orgánica } \\
(1917-1939)\end{array}$ & $\begin{array}{l}\text { Responsabilidad } \\
\text { política y representación }\end{array}$ & $\begin{array}{l}\text { Patronazgo político por } \\
\text { parte del legislativo + } \\
\text { implementación parcial } \\
\text { del servicio civil }\end{array}$ & $\begin{array}{l}\text { Partidos políticos y } \\
\text { democratización de la } \\
\text { administración pública }\end{array}$ \\
\hline $\begin{array}{l}\text { Modernización } \\
\text { administrativa } \\
(1940-1946)\end{array}$ & $\begin{array}{l}\text { Crecimiento administrativo } \\
\text { y centralización política }\end{array}$ & $\begin{array}{l}\text { Servicio civil + patronazgo } \\
\text { (PPD) }\end{array}$ & $\begin{array}{l}\text { Reducción del gobierno } \\
\text { y profesionalización del } \\
\text { servicio público }\end{array}$ \\
\hline $\begin{array}{l}\text { Reorganización } \\
\text { administrativa del } \\
\text { Ejecutivo } \\
(1947-1967)\end{array}$ & $\begin{array}{l}\text { Eficiencia (racionalización } \\
\text { de las estructuras } \\
\text { organizacionales), reducción } \\
\text { administrativa (centralización } \\
\text { de funciones administrativas) }\end{array}$ & $\begin{array}{l}\text { Servicio civil + gobierno } \\
\text { por parte de élites } \\
\text { puertorriqueñas } \\
\text { Consolidación del servicio civil, } \\
\text { modelo centralizado }\end{array}$ & $\begin{array}{l}\text { Participación ciudadana y } \\
\text { capacidad técnica }\end{array}$ \\
\hline $\begin{array}{l}\text { Bipartidismo } \\
\text { cerrado } \\
\text { (1968-presente) }\end{array}$ & $\begin{array}{l}\text { Lealtad política y crecimiento } \\
\text { administrativo }\end{array}$ & $\begin{array}{l}\text { Patronazgo político (PPD y PNP) } \\
\text { Descentralización del servicio } \\
\text { civil (2004) }\end{array}$ & Corrosión administrativa \\
\hline
\end{tabular}

Fuente: Elaboración propia con base en Santana (1994), Colón-González (2012) y Cordero-Nieves et al. (2016).

\section{CONTEXTO: EL CASO DE PUERTO RICO}

Luego de contar con un sistema de servicio civil consolidado con reconocimiento a nivel internacional, la administración de los recursos humanos en el gobierno de Puerto Rico ha sufrido un decaimiento sostenido (Santana, 1994), con el resultado de que las estructuras formales establecidas para hacer prevalecer al mérito sean manipuladas sistemáticamente por intereses político-partidistas y particulares (Comisión de Derechos Civiles, 1993; Colón-González, 2012). Se ha evidenciado el desarrollo y la persistencia de estas prácticas a lo largo del tiempo y los altos costos que estas representan (ej., Comisión de Derechos Civiles, 1993; Cordero-Nieves et al., 2016). Este deterioro se le ha atribuido en parte a la alternancia política que comenzó a finales de la década de 1960 (Santana, $1994)^{1}$ (véase el cuadro 1 ) .

\footnotetext{
${ }^{1}$ Para estudios detallados sobre la historia del servicio civil y cambios en legislación, véanse Colón-González (2012), Pacheco-Muñiz y Vera-Rodríguez (2009) y Santana (1994).
} 
La primera ley de servicio civil en Puerto Rico se adoptó en 1907, nueve años después de la ocupación estadounidense. Las leyes aprobadas en las décadas de 1930 y 1940 colocaron a las leyes de servicio civil de Puerto Rico entre las más avanzadas del mundo (Oficina del Gobernador, 1959: 136-137). Sin embargo, al igual que en otros países, la implementación inicial fue parcial, ya que Puerto Rico no contaba con la infraestructura institucional necesaria (Colón-González, 2012; Ingraham, 1995). Los avances en la implementación de las disposiciones del sistema de mérito a mediados de la década de 1940 se le atribuyeron al firme control y dominio que había logrado el Partido Popular Democrático sobre la legislatura y burocracia (Goodsell, 1965). Solo cuando la implementación del mérito dejó de ser una amenaza para el partido en el poder, se lograron avances en su implementación y consolidación (Goodsell, 1965).

Sin embargo, la tradición del mérito no fue lo suficientemente fuerte para resistir los grandes cambios políticos que ocurrieron en Puerto Rico a partir de finales de la década de 1960, cuando los dos partidos principales, el Partido Nuevo Progresista (PNP) y el Partido Popular Democrático (PPD), comenzaron a alternarse en el poder cada cuatro u ocho años. La victoria del PNP en 1968 marcó el fin de la hegemonía de veinte años del PPD, que fue seguida por un marcado aumento en la politización del servicio público en Puerto Rico durante la década de 1970 (Colón-González, 2012). El periodo posterior vio el debilitamiento de las disposiciones del sistema de mérito y una politización creciente y marcada del servicio civil en Puerto Rico (Santana, 1994; Ruiz-Acevedo, 1996). Esto, a pesar de los fallos de la Corte Suprema de Estados Unidos, que durante el mismo periodo dictó que las prácticas de patronazgo son inconstitucionales. Dentro del contexto estadounidense, Puerto Rico se considera de las jurisdicciones con más casos de discriminación política (Lopez-Quinones v. Puerto Rico Nat. Guard, 526 F.3d 23, 29 [1st Cir. 2008]), por los cuales se estima que el gobierno de Puerto Rico ha pagado aproximadamente 196 millones de dólares por década (Cordero-Nieves et al., 2016).

El debilitamiento estructural del sistema de mérito se logró a través de diversos medios, tales como reformar leyes, establecer proyectos especiales no sujetos al sistema de mérito, nombrar empleados transitorios por tiempo indefinido y luego trasladarlos al servicio clasificado mediante legislación especial y órdenes ejecutivas, uso de personal no capacitado para administrar los exámenes de ingreso, descentralización de la administración de los recursos humanos y un menosprecio por las leyes (Colón-González, 2012; Cordero-Nieves et al., 2016; Pacheco-Muñiz y Vera-Rodríguez, 2009). 


\section{REVISIÓN DE LA LITERATURA}

\section{Patronazgo}

El patronazgo se refiere a la gestión de personal con base en criterios partidistas, de corte ideológicos o de lealtad personal, en lugar de sus aptitudes o mérito profesionales (Van Riper, 1958). El patronazgo se considera una herramienta administrativa y política poderosa con múltiples funciones, como obtener y conservar el poder burocrático (Bearfield, 2009; Ozturk, 2005: 43). Como sistema de administración de recursos humanos encarna la capacidad de respuesta política y de la representatividad (Llorens et al., 2017); valores importantes en una democracia. Por ello, muchos sistemas civiles mantienen posiciones para las cuales se puede reclutar con base en preferencias, como lo puede ser la lealtad personal, política o afinidad ideológica (Peters y Pierre, 2004).

Sin embargo, al patronazgo se le ha relacionado con ineficiencia, baja calidad de la fuerza laboral y la falta de continuidad e impredecibilidad de la gestión gubernamental, lo cual afecta negativamente la confianza en las instituciones (Grindle, 2012; Ozturk, 2005). Asimismo, el patronazgo debilita los lazos entre la ciudadanía, y disminuye las inversiones en capital humano (Ozturk, 2005: 31; Ingraham, 1995). Por otro lado, este sistema ha sido vinculado a la corrupción pública, y sus prácticas se consideran mecanismos que facilitan la corrupción sistémica con enormes repercusiones sociales, políticas y económicas (Heidenheimer et al., 1989; Oliveros, 2013; Ozturk, 2005; Meza y Pérez-Chiqués, 2020).

En la literatura, el patronazgo se ha caracterizado como propio de las primeras fases de la democratización, y se le ha relacionado con periodos de agitación y cambio social, como lo son cambios de régimen, cambios en el orden económico o en los patrones de acceso y exclusión a la influencia política y a los servicios gubernamentales (Heidenheimer et al., 1989; Klingner, 1996; Scott, 1969). Por ejemplo, en América Latina se ha visto un afianzamiento de las prácticas clientelares a partir de las transiciones democráticas en la región (Ippolito-O'Donnell, 2006: 2). La mayoría de los países latinoamericanos aprobaron sus primeras leyes de servicio civil al menos cincuenta años después de Puerto Rico, algunos hasta en las décadas de 2000. Al igual que en Puerto Rico, en muchos de estos países existe una desconexión marcada entre el sistema formal e informal de gestión de personal, o una distancia entre las normas y prácticas (Iacoviello y Zuvanic, 2006).

Aunque el patronazgo se considera una práctica que puede superarse o moderarse una vez que cambien las condiciones que lo originaron y otros métodos de influencia o intercambio político estén disponibles o se vayan arraigando (Johnston, 1979; Scott, 1969; Llorens et al., 2017), la experiencia de Puerto Rico, así como de otros 
países donde ha aumentado la politización (Cooper, 2020), sugiere que las trayectorias en la administración de los recursos humanos en el sector público no son tan predecibles (ej., Klingner, 1996). Por lo tanto, es necesario entender mejor las modalidades en las que se esté abusando del poder público mediante los sistemas de administración de recursos humanos.

\section{Servicio civil neutral como remedio}

En contraposición a los sistemas de patronazgo, el establecimiento de un servicio civil neutral y profesional basado en el mérito fue impulsado como una medida para erradicar prácticas patrimonialistas — como el sistema de botín-y establecer una clara división entre la administración pública y la política (ej., Wilson, 1881). En ese sentido, los servicios civiles se han impulsado como pieza fundamental de las administraciones públicas modernas, ya que se consideran como propulsores de profesionalismo, autonomía y neutralidad burocráticas, y abonan al buen funcionamiento, continuidad y previsibilidad del gobierno (ej., Martínez Puón, 2006; Llorens et al., 2017; Dahlström et al., 2012).

Una finalidad de estos sistemas es poder garantizar la continuidad de programas y políticas a través de la estabilidad laboral ante los cambios de gobierno (Martínez Puón, 2006). Para lograr esto, el servicio civil cuenta con un valor preponderante que es la neutralidad política, la cual se refiere, paradójicamente, a la falta de valor, pero "que es el estándar contra el cual los servidores públicos son juzgados" (Box, 2015: 51). A lo largo de la historia, este ha sido un valor complejo de alcanzar, por lo que Box (2015) lo describe como un "ideal”, ya que las esferas de la política y la administración pública difícilmente pueden separarse. De esta manera, la forma en la que los servidores públicos son empleados por el gobierno tiene un impacto crucial para la democracia (Geddes, 1994). Por lo tanto, será parcial aquel servidor público que ingrese al servicio mediante una designación política, mientras que, en contraparte, será neutral aquel que ingrese por méritos profesionales.

En ese mismo sentido, el servicio civil es considerado un tipo de protección contra el patronazgo, ya que aminora o imposibilita otras prácticas consideradas corruptas (ej., Charron et al., 2017; Grindle, 2012), y fomenta la transparencia y la rendición de cuentas (ej., Oliveros y Schuster, 2018; Grindle, 2012). Contrario a los sistemas patrimoniales, en los sistemas de servicio civil los empleados son agentes del Estado y no de un partido o de un patrono, así se fomenta un acceso al servicio público basado en criterios universalistas, y no particularistas o personalistas (ej., Oliveros, 2016). 
Los estudios sobre la politización de la burocracia sostienen que el patronazgo debería disminuir en la medida en que los partidos introduzcan sistemas de gestión de recursos humanos basados en el mérito para evitar los nombramientos políticos cuando algún partido de la oposición llegue a ocupar el gobierno (Geddes, 1994; O’Dwyer, 2006). Así, los gobiernos han apostado a los sistemas de servicio civil como una alternativa o, incluso, como la solución ante los sistemas de botín y prácticas de patronazgo (Bearfield, 2009). Como señala Grindle (2012: 19), las expectativas en cuanto a las promesas de las reformas de servicio civil han sido inmensas, se ha visto como una medida contra "la corrupción, el nepotismo, el favoritismo, partidismo, despojo ('spoils'), incompetencia, falta de profesionalismo, inequidad, captura, particularismo, mediocridad, malversación y el fraude electoral y la violencia”. Sin embargo, como señala la autora, estas expectativas no se han cumplido en muchos lugares, incluyendo muchos países latinoamericanos (ej., Kearney, 1986; Nieto y Pardo, 2019; Schuster, 2017).

Es preciso señalar que las repetidas fallas en la implementación de los servicios civiles deben alertar sobre la necesidad de entender mejor las dinámicas que previenen su consolidación (ej., Schuster, 2016; 2017) o desembocan en su desarticulación (ej., Pacheco-Muñiz y Vera-Rodríguez, 2009). En el caso del servicio civil en Puerto Rico, por ejemplo, este se ha enfrentado a una constante adaptación de la realidad democrática en la que no ha podido ser separada la administración pública de la política partidista. Para asegurar que las burocracias sean actores de contrapeso y neutrales, es necesario que se impulse al mérito para fortalecer las estructuras formales burocráticas y, con ello, mejorar la calidad de la democracia y la provisión de los servicios públicos.

\section{Neopatrimonialismo}

Este artículo basa su análisis en la noción de neopatrimonialismo —o patrimonialismo moderno- (Eisenstadt, 1973) para examinar los efectos del patronazgo en la gestión de recursos humanos en la administración pública en Puerto Rico. Aunque gran parte de los análisis realizados con esta aproximación teórica se han enfocado recientemente en el estudio de sistemas políticos en países africanos (Erdmann y Engel, 2007), algunos autores han utilizado este marco teórico para analizar sistemas administrativos y políticos en América Latina (por ej., Durazo, 2010; Oszlak, 1986; Remmer, 1989) que se caracterizan por contar con regímenes con incipientes instituciones democráticas y burocracias estatales débiles (Erdmann, 2002).

El neopatrimonialismo es un enfoque analítico que permite explicar los procesos de hibridación entre las reglas formales e informales en sistemas políticos y admi- 
nistrativos. Esta categoría analítica se utilizará para describir las formas en las que los partidos políticos se han apoderado sistemáticamente del servicio civil en Puerto Rico a través de prácticas informales, convirtiendo al servicio civil en un instrumento de legitimación en beneficio del partido político en el gobierno en turno.

El neopatrimonialismo se deriva de la concepción weberiana del patrimonialismo. Para Max Weber (2002), el patrimonialismo representa una de las formas de dominación tradicional donde el soberano que ostenta el poder se legitima por órdenes y poderes heredados en forma de santidad desde tiempos inmemoriales. Aquí, las relaciones del cuadro administrativo no se establecen por el deber objetivo del cargo sino por la fidelidad personal del servidor hacia el soberano (Weber, 2002: 180).

La característica esencial del patrimonialismo es la falta de distinción entre las esferas pública y privada de la sociedad, siendo ambas propiedad del gobernante como fuente de riqueza personal (Weber, 2002). Según Roth (1968), el patrimonialismo no existe más en los Estados "modernos" debido a la paulatina desaparición de los regímenes patrimoniales, aunque sus modos de operación y arreglos administrativos persisten, lo que justifica la aplicación de este marco teórico a los sistemas modernos. En ese sentido, Eisenstadt (1973) acuña el término neopatrimonialismo para analizar estos sistemas (patronazgo, clientelismo, autoritarismo), que representan un desarrollo desequilibrado entre lo "moderno" y lo "atrasado" dentro de un mismo territorio. El neopatrimonialismo describe una forma de relación política que se basa en mecanismos de dominación tradicional en el marco de un sistema legal-burocrático (Monsiváis y del Río, 2013), y ha servido para explicar sistemas administrativos y políticos donde la autoridad establecida de manera formal en un Estado moderno (por ej., servicio civil) no ha logrado terminar con las formas tradicionales de dominación política (por ej., patronazgo) (Erdmann y Engel, 2007).

Esta categoría analítica ha permitido estudiar la existencia de fenómenos compuestos por tendencias en conflicto que, a pesar de sus contradicciones, logran adaptarse y comprenderse (Monsiváis y del Río, 2013), como lo es el caso de Puerto Rico. Aquí, el neopatrimonialismo se ha asentado como la forma en la que los partidos políticos, mediante la integración de prácticas de patronazgo (dominación tradicional), se ha apoderado del servicio público manteniendo al servicio civil (dominación legal-racional) solo como una "fachada" funcional en la que los servidores públicos se ven forzados a mostrar lealtad en beneficio del partido "soberano". La hibridación de estos dos sistemas ha provocado que la distinción entre lo público y lo privado sea difusa, lo que permite a los partidos 
políticos apropiarse del servicio público y sus instituciones de manera estratégica y discrecional (Pérez-Chiqués y Rubin, 2021).

La literatura del servicio civil en América Latina ha evidenciado que la poca consolidación de los servicios civiles en la región se debe, en gran parte, a la politización de las estructuras formales (por ej., Argentina [Chudnovsky y Cafarelli, 2018]; Chile [Fuenzalida y Riccucci, 2019]; Colombia [Puentes, 2017]; México [Pardo, 2005]; Perú y República Dominicana [Ramió, 2008]). Esta politización se ha desarrollado con el objetivo de poder gestionar los recursos humanos mediante estrategias destinadas a "burlar" la ley y los principios rectores del sistema. En ese sentido, se espera que en sistemas híbridos de gestión de recursos humanos, donde las prácticas patrimonialistas estén más arraigadas y desarrolladas que las prácticas formales, los servidores públicos que fueron contratados por méritos profesionales sean condicionados por sus patrones políticos para seguir alimentando la maquinaria del patronazgo. El caso de Puerto Rico, un contexto latinoamericano dentro del contexto político-institucional estadounidense, da luz sobre las prácticas neopatrimonialistas que pueden replicarse en otros sistemas de servicio civil de la región, cuyas características de debilidad institucional y burocrática son una constante.

\section{METODOLOGÍA}

Con el fin de identificar y entender las estructuras y prácticas informales en la administración de los recursos humanos en las agencias del gobierno de Puerto Rico, así como la experiencia de los empleados gubernamentales en dicho sistema, fueron llevadas a cabo 29 entrevistas con 23 empleados públicos. El perfil que se perseguía para las personas entrevistadas era que $a$ ) hubiesen ocupado un puesto de carrera en una agencia ejecutiva del gobierno de Puerto Rico y $b$ ) hubiesen vivido al menos un cambio de gobierno trabajando en una agencia. Se buscó también identificar a personas con conocimiento directo de las prácticas de recursos humanos y de las prácticas de los grupos políticos dentro de las agencias (ej., empleados de confianza). Adicionalmente, se procuró tener balance en la afiliación política de las personas entrevistadas. Tanto las entrevistas (estrategia central) como el análisis documental (estrategia complementaria) tenían la finalidad de identificar patrones en la administración de recursos humanos (formales e informales); entender el rol de la afiliación política en la trayectoria profesional de los empleados de carrera; identificar mecanismos para la inclusión de la afiliación política en procesos de recursos humanos, incluyendo los instrumentos utilizados, y los actores involucrados, y entender la perspectiva de los empleados públicos y 
el aprendizaje que resulta de sus experiencias. Con esta aproximación, la expectativa era lograr obtener un cuadro tanto de las prácticas informales como de la experiencia de los empleados.

Las entrevistas comenzaron en diciembre de 2014 y finalizaron en agosto de 2017. Además, se realizaron entrevistas de seguimiento con seis de las personas entrevistadas durante el periodo 2014-2015, luego de las elecciones de 2016, que resultó en un cambio del partido político en el poder. Véase gráfica 1 para contar con mayor referencia acerca de la evolución en el número de puestos ocupados mediante el servicio civil en los últimos años.

La muestra $(\mathrm{N}=23)$ incluyó a personas con experiencia laboral en el gobierno a nivel estatal y municipal. Los servidores entrevistados trabajaron durante su carrera en áreas gerenciales, administrativas, de servicio directo y de recursos humanos. Todos ellos ocuparon puestos de carrera durante su trayectoria laboral - doce también ejercieron en puestos de confianza, y seis trabajaron en calidad de contratistas para el gobierno-. En cuanto a su antigüedad laboral: dos habían trabajado menos de quince ańos, quince habían trabajado entre quince y treinta años, y seis contaban con más de treinta años de experiencia (dos estaban jubilados).

Las entrevistas en profundidad duraron un promedio de 1.5 horas y las preguntas se centraron en las experiencias de los empleados, en cómo entraron a trabajar al gobierno, sus experiencias durante periodos de alternancia política, el rol o

GRÁfICA 1. Personal empleado en el gobierno de Puerto Rico, 2008-2021

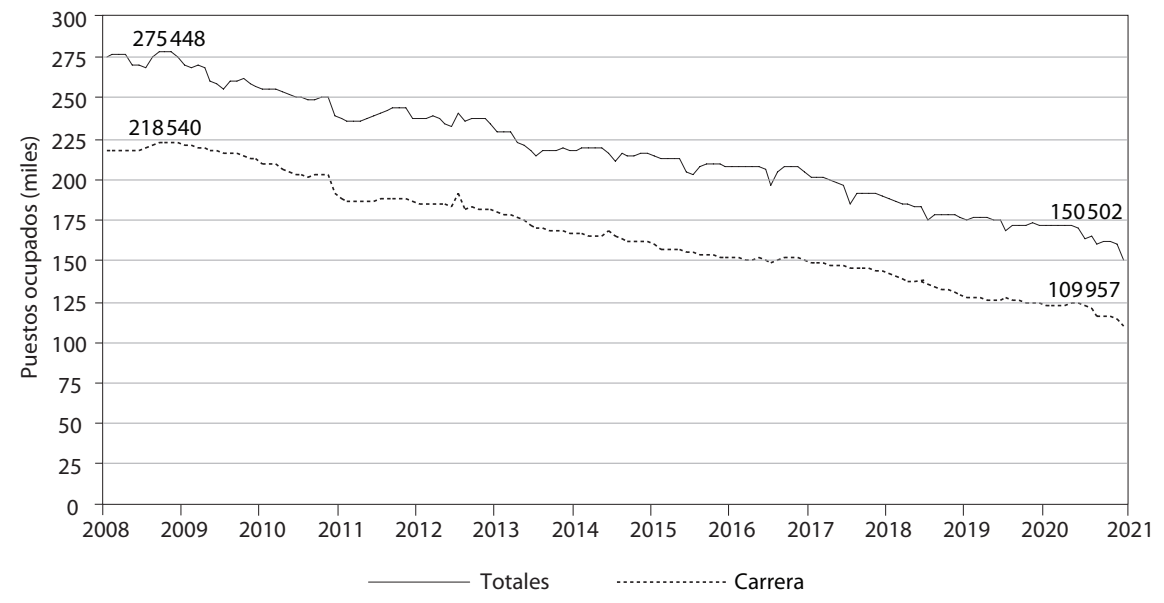

Fuente: Instituto de Estadísticas de Puerto Rico (2021). 
CUAdRo 2. Personal empleado en el gobierno de Puerto Rico por categoría, 2021

\begin{tabular}{|c|c|c|c|c|c|}
\hline \multirow[b]{2}{*}{ Carrera } & \multicolumn{2}{|c|}{$\begin{array}{l}\text { Total administración } \\
\text { pública } 2021 \text { (AP) }\end{array}$} & \multicolumn{2}{|c|}{$\begin{array}{c}\text { Departamentos y } \\
\text { agencias } 2021 \text { (DA) }\end{array}$} & \multirow{2}{*}{$\begin{array}{c}\text { Porcentaje } \\
\text { DA / AP (\%) } \\
65.95\end{array}$} \\
\hline & 109957 & $73.06 \%$ & 72519 & $87.93 \%$ & \\
\hline Confianza & 6790 & $4.51 \%$ & 1377 & $1.67 \%$ & 20.27 \\
\hline Otros* & 33755 & $22.43 \%$ & 8576 & $10.40 \%$ & 25.41 \\
\hline Total & 150502 & $100.00 \%$ & 82472 & $100.00 \%$ & 54.80 \\
\hline
\end{tabular}

Fuente: Elaboración propia con base en Instituto de Estadísticas de Puerto Rico (2021). *Se refiere a otros puestos transitorios o irregulares.

relevancia de la afiliación política y del mérito en su día a día, y su trayectoria profesional. Adicionalmente, se les preguntó acerca de sus experiencias de discriminación política y de la intromisión de la política partidista en sus trabajos.

Todos los entrevistados trabajaron en departamentos y agencias del Estado, que cuentan con los sistemas más robustos de administración de recursos humanos, y con la fuerza laboral más profesionalizada en el gobierno de Puerto Rico (Cordero-Nieves, 2014). Para el ańo 2021, del total de los puestos de carrera en todo el gobierno, 65.95 por ciento pertenece a este tipo de agencias (cuadro 2). Asimismo, cabe señalar que los dos partidos políticos principales estuvieron representados en la muestra: once entrevistados estaban afiliados al PPD, nueve estaban afiliados al PNP, y tres de los entrevistados no revelaron su afiliación política.

Las entrevistas fueron transcritas y codificadas utilizando un acercamiento inductivo (Emerson et al., 1995). La codificación abierta sirvió para identificar conceptos iniciales que dirigieron las rondas subsiguientes de codificación, las cuales estuvieron centradas en analizar tendencias en cuanto a la experiencia de los empleados (su reclutamiento, experiencias luego de un evento electoral y cambio de administración, experiencias de discriminación política), prácticas informales y conceptos relacionados (confianza, desconfianza, grupos políticos, listas), involucramiento de las oficinas de recursos humanos en prácticas discriminatorias, entre otras. Se utilizó la herramienta Atlas.ti para apoyar la codificación y el análisis.

Para complementar las entrevistas, confirmar patrones, y adquirir información adicional sobre los mecanismos y los instrumentos utilizados para la discriminación política en el empleo público se analizaron cincuenta casos de discriminación política que involucran a empleados públicos seleccionados al azar. Para esto, se 
utilizó la base de datos de Westlaw para la selección de los casos, la cual permite el acceso a expedientes en las cortes federales y estatales donde se atienden los casos de discriminación política en Puerto Rico. Los casos proporcionan un registro de cómo se experimenta el sistema de patronazgo informal. Los documentos se subieron al programa Atlas.ti y se siguió el mismo esquema de codificación desarrollado para las entrevistas: experiencia de los empleados, prácticas informales en la administración de los recursos humanos y actores involucrados. El análisis de estos casos ayudó a confirmar tendencias, además de conseguir evidencia de prácticas mencionadas por las personas entrevistadas.

Por último, se analizó en profundidad el expediente de otros doce casos de discriminación política utilizando el sistema de Acceso Público a Registros Judiciales Electrónicos. Las transcripciones judiciales, declaraciones y las pruebas de los casos seleccionados mostraron evidencia referente al trabajo de los grupos políticos que laboran dentro de las agencias estatales, su trabajo de vigilancia política y el rol del personal de recursos humanos en apoyo a objetivos políticamente discriminatorios. En lugar de ofrecer un análisis sobre la implementación de los diferentes subsistemas del servicio civil en Puerto Rico, este artículo resalta estructuras y prácticas informales que surgieron como principales en el proceso de análisis.

\section{RESULTADOS}

El caso de Puerto Rico es uno de resurgimiento del patronazgo dentro de las estructuras formales del servicio civil. Es un sistema de patronazgo informal que está limitado por las restricciones que le impone el sistema racional-legal, pero que continúa funcionando a través de los instrumentos designados como esenciales al principio de mérito (ej., reclutamiento y selección; ascensos/descensos, traslados, procesos disciplinarios y despidos). Esta sección proporciona una descripción y análisis de la infraestructura y las herramientas del sistema informal existente, que subyacen, refuerzan y dan forma a la experiencia del patronazgo.

\section{Experiencia mediada por ciclos políticos}

La inclusión sistémica del criterio político en los procesos de reclutamiento y selección de empleados de carrera, así como las prácticas discriminatorias dirigidas a empleados de carrera que no son del partido en el poder, han resultado en un servicio de carrera de carácter permanente politizado que se divide en gran medida entre los dos principales partidos políticos, el PNP y el PPD; el partido que tenga el mayor número de militantes en el sistema de carrera, es el que tiene mayor control y mayor poder. Como explicó un testigo en una vista pública, 
Lamentablemente, aquí en Puerto Rico, mientras hubo monopolio político por el Partido Popular, pues no había problemas. Cuando gana el Partido Nuevo Progresista por primera vez, pues pusieron supervisores para que supervisaran a los que sabían del Partido Popular. Gana el Partido Popular otra vez, entonces pone un supervisor para supervisar al que supervisaba del PNP al del Partido Popular. Y en ese proceso de esos supervisores se perdió esa carrera política de los que van subiendo (Senado de Puerto Rico, 2016: 117).

De hecho, de las entrevistas y casos judiciales, se desprende que la fecha de entrada al sistema de empleo público se utiliza como proxy informal para determinar la afiliación política de los empleados de carrera, y, esta información, a su vez, se utiliza para tomar decisiones de recursos humanos.

De los 23 entrevistados, 21 expresaron haber conseguido sus plazas por medio de "palas" - término coloquial que refiere a conexiones personales, familiares o políticas- Todos los entrevistados describieron la manipulación extensa de los procesos de contratación para ingresar personas afines al partido; además de prácticas como la utilización de "cartas de recomendación" de personas conectadas políticamente; la simulación de concursos abiertos en la contratación de personal de carrera para la contratación de personas preelegidas ("ese puesto tiene nombre y apellido"); así como la manipulación de los puestos transitorios para evadir requisitos del servicio de carrera (véase también, Colón-González, 2012; CorderoNieves et al., 2016). Además, todos los entrevistados expresaron que su carrera había sido en gran parte determinada por su afiliación política o por la afiliación que se les atribuyó. Es decir, sus oportunidades y condiciones laborales fluctuaron según los ciclos electorales, tendiendo a ser positiva cuando su afiliación coincide con la del partido en poder y negativa cuando no coincide.

Del análisis de casos y entrevistas se desprende que luego de un evento electoral las condiciones laborales de empleados que no son del partido vencedor pueden cambiar negativamente y, en consecuencia, pueden sufrir acoso político y hasta pérdida de empleo, variaciones de discriminación política:

En Puerto Rico, un cambio entre el Partido Popular Democrático y el Partido Nuevo Progresista [llevan a] operadores políticos fervientes del partido vencedor a despedir, descender, o reducir los salarios de los empleados afiliados al partido de oposición saliente (LópezQuinones v. Puerto Rico Nat. Guard, 2008).

Entre las prácticas de discriminación política más comunes según la muestra de casos están: el hostigamiento o acoso por motivos políticos, los despidos constructivos o establecer condiciones intolerables para que las personas renuncien, 
los despidos y las transferencias punitivas. Estos patrones muestran gran continuidad a las prácticas de discriminación política señaladas por la Comisión de Servicio Civil de Puerto Rico en 1993 (Comisión de Gobierno, 2006).

Como describió una empleada de carrera, "después de un cambio de administración, todo cambia, la gente cambia”. Los patrones generales identificados en las entrevistas y los casos judiciales apuntan a una experiencia colectiva marcada por los ciclos electorales. Los empleados de carrera pasan por periodos de ser favorecidos y gozar de confianza por su afiliación política - que usualmente coincide con el periodo en que fueron reclutados - a ser discriminados o tratados con desconfianza, cuando hay un cambio de partido en el poder (véase también, Pérez-Chiqués y Rubin, 2021). Debido a que las protecciones del servicio civil sí han limitado los despidos masivos, los empleados favorecidos y discriminados coexisten en ambientes donde el estatus de los empleados es dictado por su afiliación política. Los empleados presencian cómo varían las condiciones laborales de sus compañeros de trabajo de acuerdo con el partido que está en poder, lo cual afecta y cambia las dinámicas grupales entre los empleados y resulta en un aprendizaje importante para estos sobre lo que requiere el sistema para ascender. La carrera se hace con el partido, como expresó una exempleada de carrera, "tienes que apostarle tu carrera a un partido, y esperar que así puedas subir profesionalmente". Como explicó otro exempleado de carrera:

Así es como funciona este mundo y es frustrante para uno, porque uno es un trabajador, uno trata de entrar sin fines políticos y al fin y al cabo termina definiéndose políticamente porque no te queda de otra. O que ellos mismos te obligan. ... Cuando le digo que te obligan y te llevan a eso, es que como tú estás tratando de ganarte el pan con tu gente, dentro de lo que viene siendo la parte profesional, sin cuestiones políticas, te das cuenta que estás en una encrucijada donde dices, bueno, si soy rojo los azules me van a matar y si soy azul, los rojos me van a matar también. Pero si soy ambiguo, tampoco ninguno de los dos me da trabajo. Entonces te ves obligado a definirte con los valores y principios políticos, a definirte para poder trabajar con el gobierno.

Los empleados en este sistema aprenden que la neutralidad es vista con desconfianza y que la parcialidad política es importante para conseguir un empleo público, mantenerlo y desarrollarse profesionalmente. Además, crean la expectativa de que serán favorecidos o discriminados de acuerdo con el partido que esté en el poder y actúan conforme a esta expectativa. Es un sistema que promueve una división de la fuerza laboral en función de su afiliación política, creando un ambiente que propicia una hipervigilancia sobre esta afiliación, 
Yo creo que todo el mundo está tratando de definir cuál es la afiliación política de unos y otros. Creo que en el gobierno hay una separación de amistades en función de eso; muchas de las relaciones personales se dan en función de que somos o no de un partido, es tema de conversación, es tema de pelea es tema de celebración ... principalmente entre los empleados de carrera, es parte de la diversión hablar de política y, como los nenes en la escuela cuando dicen, cuando pelean por algún deporte o por alguna razón, la que sea. ... "yo voy a estar mejor que tú porque está este partido". O "espera que pierdan para que tú veas lo que te vamos a hacer", ese tipo de cosa (empleada de carrera).

Los patrones de administrar (informalmente) al personal en el servicio de carrera con base en su afiliación política abona a la construcción de la confianza y de desconfianza basados en el criterio político, en lugar de otro factor, como el profesionalismo o el mérito. Esta construcción de la confianza y la desconfianza subyace a la manera en que se establecen jerarquías informales dentro de las agencias que responden a los partidos políticos,

[...] el componente del partido político se da en todos los niveles de la organización, desde las oficinas locales, hasta la oficina central. Desde puestos que puedan estar en las primeras escalas salariales como oficinistas, conserjes o secretarias, hasta los puestos que están en las escalas altas como los especialistas o los directores de finanzas y presupuesto. Pasando por cualquier personal técnico, compras y demás. Así que cada cual en su área pues tiene un quantum de poder que puede ser mayor o menor, pero que es el quantum que me toca o el que tengo. Y dentro de esa jerarquía [...] algunos a lo mejor le hacen compras o le emiten los cheques a las personas del partido versus que los de otro nivel adjudican contratos, de millones de dólares, a personas del partido (empleada de carrera).

Los empleados aprenden también a discriminar con base en el criterio político, y a ver esta discriminación como funcional y necesaria para reclutar empleados de carrera que respondan a las directrices que se les impartan. Por ejemplo, si se nombra a alguien que no es del partido,

no se deben a nadie $[\ldots]$ a la hora de repartir los regalos de Reyes [...] les dio conjuntivitis, artritis, se nota que te están boicoteando y tú con la cara de pendejo. Tienen derechos, pero los usan para joderte [...] Así está montado ese andamiaje. Así sobrevive (empleada de carrera).

Como explicó una empleada de confianza, al referirse a estos patrones, "todo el andamiaje gubernamental está montado en eso. No te puedo decir que es mínimo. Está montando en eso.” 
Las prácticas descritas en esta sección, como la utilización de las “palas” (ej., Arellano Gault y Castillo Salas, 2019; Lomnitz, 1988; Jancsics, 2009), la importancia del concepto de la "confianza" en la contratación de personal (ej., Meza y Pérez-Chiqués, 2020; Chudnovsky, 2017), el efecto de los ciclos políticos en el favoritismo y la discriminación política (ej., Iacoviello y Zuvanic, 2016), la simulación o manipulación de procesos formalmente meritocráticos (ej., Dussauge Laguna, 2011; Kearney, 1986) están ampliamente documentados en el contexto latinoamericano.

\section{INFRAESTRUCTURA INFORMAL}

La mayoría de las prácticas de discriminación, o bien de favoritismo, presuponen el conocimiento de la afiliación política de los empleados. Esta sección se centrará en analizar algunas de las estructuras informales que realizan esa labor de vigilancia y que contribuyen a la sistematización de las prácticas de dominación del patronazgo.

Las estructuras informales del patronazgo movilizan la desconfianza como una estrategia para ejercer el poder y mantener el control sobre la estructura formal de la burocracia, y contribuyen a crear un sistema incongruente para los empleados de carrera, donde de manera oficial se promulga el mérito, pero que los somete a una administración de personal que se rige informalmente por criterios político-partidistas. Estas experiencias, a su vez, promueven un aprendizaje muy específico en los empleados que refuerza una lógica politizada, contraria al principio del mérito.

\section{Los grupos políticos}

Uno de los factores que sustenta a las maquinarias políticas es la capacidad de los partidos para vigilar las actividades políticas de los votantes y recompensarlos o castigarlos, dependiendo de su comportamiento político (Stokes, 2005). Esta vigilancia se consigue a través de organizaciones políticas de empleados públicos que operan informalmente dentro de las agencias de gobierno (Cordero-Nieves, et al., 2016; O’Connell v. Marrero Recio, 2013; Torres-Heredia, et al. v. LópezPena et al., 2008; Senado de Puerto Rico, 2016). Estas organizaciones están involucradas en recaudar fondos para los partidos políticos, pero también cumplen una importante función informal en la administración de los recursos humanos en las agencias. A pesar de que estas organizaciones recaudan importantes cantidades de dinero para los partidos, se conoce poco sobre su manera de operar (Cordero-Nieves et al., 2016). 
De acuerdo con las personas entrevistadas, cada partido político tiene una organización, con una directiva electa por los activistas del partido en cada agencia gubernamental (véase también, Cordero-Nieves et al., 2016). A cada agencia (el jefe de la agencia y a los grupos políticos) se le asigna una cuota de dinero que debe recaudar para el partido. A veces, recolectan este dinero legalmente, vendiendo boletos de rifa fuera de las instalaciones y fuera de horas laborables, pero algunas de estas actividades de recaudación de dinero son ilegales y coercitivas. Estas estructuras políticas dentro de las agencias públicas producen estructuras de autoridad muy complejas. Un entrevistado con conocimiento directo de la estructura política explicó cómo el poder dentro de las agencias no necesariamente coincidía con los puestos oficiales de los empleados:

No pienses en puestos de confianza, puede ser hasta el oficinista, puede ser hasta el conductor. Pero el conductor es el que tiene el poder porque es el que tiene los contactos, es el que conoce o el que le trabajó y le corrió la campaña a fulano, o tiene el poder adquisitivo para poder llegar, es el que aportó. Aunque esté en un puesto de chofer resulta [...] que aportó o recaudó suficientes fondos, pues esa persona crea como sus afiliaciones.

Continuó explicando el poder de las personas en estos grupos:

Porque acuérdate que son recaudadores de fondos y, algunas veces, estas personas de esta directiva, no es que son recaudadores de fondos porque son parte de la directiva, es porque recaudó fondos de hace mucho tiempo. O sea, son personas que dentro de la política son conocidas, a lo mejor es líder de barrio y, aunque usted no lo crea, un líder de barrio tiene un poder que Dios cuide [... ¿ ¿No ves que es el líder de barrio quien moviliza a la gente a la hora de los votos?

Los miembros de estos grupos políticos obtienen su poder no de la autoridad posicional derivada de los puestos que ocupan en las agencias, sino de su posición como recaudadores de fondos y movilizadores dentro de los partidos. Entre las actividades que realizan estos grupos, está la de vigilancia política.

\section{Las listas}

Estas organizaciones participan en la elaboración de listas de empleados que detallan su afiliación política y que se utilizan como una herramienta para decidir a quién favorecer, a quién exigir servicios políticos y contribuciones, y para identificar y discriminar en contra de personas afiliadas al partido de la oposición (Cordero-Nieves et al., 2016; Colón-González, 2012; Cordero-Nieves, 2012a, 2012b). 
En cada agencia hay un grupo de personas que son de la base del partido, verdad, que se ocupan de identificar y acercarse a las personas que van a estar aportando, de mantener una cohesión de equipo, de invitar a actividades políticas [...] difundir los mensajes del partido [...] Son los que organizan a las personas, los que recaudan dinero, los que amenazan a los que no pagan, es como una mafia [...] Es oficial en el sentido de que cada partido tiene su organización, y dentro de su organización, estas personas pertenecen a esos comités. No es oficial en el sentido de que no está sancionado por el empleador [...] se sale de los límites oficiales $[\ldots]$ (empleada de carrera).

Un empleado de carrera que participa de estos grupos explicó:

El presidente (del grupo político de la agencia) nombra a una persona en la agencia para mantener la lista, para identificar quién es quién, popular o penepé, ${ }^{2}$ en toda la agencia. [¿̨Para qué se usa la lista?] Para pedirles dinero, para pedirles que vendan boletos [...] [iंY si dicen que no?] Después no pueden venir a pedir ayuda. [¿Qué tipo de ayuda?] Ayuda como para conseguir trabajo a un familiar, acomodar [en un puesto] a alguien que ellos conocen, ese tipo de cosas.

Existen listas oficiales de personas que cualifican por mérito para ocupar ciertos puestos, así como estas listas no oficiales de personas que cualifican debido a su lealtad política. Estas listas, mencionadas en nueve casos judiciales ${ }^{3}$ y en múltiples entrevistas, funcionan como listas formales basadas en méritos, determinando contrataciones, promociones y despidos. Estas listas a veces se envían desde los partidos y otras veces son elaboradas por los grupos en las agencias. Como evidencian los casos y las entrevistas, estos grupos trabajan de la mano con el personal de confianza y con las oficinas de recursos humanos.

Como explicó una persona entrevistada, cuando asumió por primera vez un puesto de confianza, empleados del grupo político le dieron dos listas de empleados de carrera. Le dijeron: "estas son las personas que trabajarán contigo y te apoyarán,

2 "Popular" se refiere a los miembros del Partido Popular Democrático, y "penepé" a los pertenecientes al Partido Nuevo Progresista.

${ }^{3}$ Alvarez-Estrada v. Alemany-Noriega, Not Reported in F.Supp.2d (2011); Diaz-Garcia v. SurilloRuiz,113 F.Supp.3d 494 (D.P.R. 2015); O’Connell vs. Marrero Recio, CASE 3:10-CV-01971-MEL, Document 89-2, Filed 05/03/12; Pagan-Cuebas, et al. v. Vera-Monroig, et al., 91 F. Supp. $2 d 464$ (D.P.R. 2000); Peguero-Moronta v. Santiago, 464 F.3d 29 (First Cir., 2006); Franco-Figueroa v. State Ins. Fund, No. CIV 11-1025 JAF, 2013 WL 4434252, at *4 (D.P.R. 2013); Ocasio-Hernandez v. Fortuno-Burset, 639 F. Supp.2d 217 (2009); Torres-Heredia et al. v. Lopez-Pena et al., No. CIV 02-1466 (2008), 708 F.Supp. $2 d$ 148 (D.P.R. 2008); Santiago-González et al., v. Banco de Desarrollo Económico de Puerto Rico et al., CIV No. 01-1865 (JAF) (2001). 
y estas son las personas que no lo harán”. Aunque al principio no estuvo de acuerdo con esa distinción, y quería trabajar con todos, "sin importar colores," con el tiempo considera que estas listas son herramientas útiles para administrar al personal, y ha adaptado criterios similares en los procesos de contrataciones para el personal de carrera:

Mi mejor empleado es uno que es "de confianza" y "confiable". Por "de confianza" quiero decir que militan en mi partido [...] que se puede hablar de todo con ellos, que me son incondicionales, que puedo contar con ellos para ejecutar la política pública del partido [...] Por "confiable" me refiero a que lleguen a trabajar a tiempo, que no causen problemas con otros empleados [...] Prefiero dejar el puesto desocupado que contratar a una persona que, aunque confiable, no es de confianza.

Esto fue un patrón común entre personas que entraron inicialmente al empleo público en puestos de confianza. Aunque en un principio no les importaba "quién era quién", ahora consideran que las cualificaciones políticas, que el "ser de confianza" es indispensable para las contrataciones de personal de carrera.

Hay variaciones en el tipo de listas de afiliación política y en el uso que se les da a las listas. Además de ser utilizadas como una herramienta en la selección de empleados, son utilizadas como herramienta administrativa (saber con quién contar en el grupo de trabajo o con quién tener cuidado), para la toma de decisiones (por ej., a quién proteger de recortes al personal [O'Connell vs. Marrero Recio, 2012]), y, para hacerle daño a personas específicas, usualmente, por "revanchismo". Como explicó una persona entrevistada, "es una manera de humillar, de cobrarse cosas."

Un tipo de lista mencionada por los entrevistados y en algunos casos judiciales, son las "listas negras", o listas de empleados que son identificados porque son de otro partido político o porque han caído en desgracia con el grupo. Estas listas se usan explícitamente para tomar decisiones adversas o para hacer daño (despidos, descensos, acoso).

Por ejemplo, en un caso de discriminación política, un testigo declaró en su deposición que "hubo una reunión el 19 de diciembre de 1999 en el Comité Central del Partido Popular Democrático (PPD) en la que se dieron instrucciones de que identificáramos a los empleados del Partido Nuevo Progresista (PNP) para sustituirlos con miembros y activistas del PPD" (Torres-Heredia v. López-Pena, 2008). El coacusado que ordenó la lista, a la que se refirió como "la lista negra", era el presidente del grupo de empleados del PPD en la corporación pública y también era el director de Recursos Humanos de la corporación pública. En otro 
ejemplo, las instrucciones para preparar "listas negras" venían de funcionarios de alto nivel en las agencias:

Los directores de las oficinas locales por instrucción del [imputado] Wilfredo Alemañy [el Secretario de la Familia] [...] comenzaron a hacer listas de todos los empleados que fueron identificados con el PPD y tenían que ser despedidos [...] Alemañy se reunió con varios empleados del pNP [...] para discutir los despidos [...] Alemańy acosó a los empleados que habían sido notificados de su despido burlándose de ellos y les preguntó "oye, ¿̇no te despedí ya?” Alrededor de enero o febrero de 2009, Alemañy solicitó a un empleado del Departamento de la Familia [...] que preparara una lista de todo el personal que no era miembro del pNp. Cuando ella se negó, la lista fue preparada por Wanda Pabón. Alemañy preparó una carta a Recursos Humanos informando qué puestos quería eliminar (Álvarez-Estrada v. Alemany-Noriega, 2011; traducido por autores).

Como ilustran estos ejemplos, los grupos políticos de empleados públicos y las listas que elaboran o socializan son componentes importantes del sistema informal de administración de recursos humanos en el gobierno de Puerto Rico, que contribuyen a politizar a los empleados públicos y a que los partidos puedan ejercer mayor control sobre los empleados y las burocracias.

\section{CONCLUSIÓN}

Uno de los principales objetivos del servicio civil es fortalecer un aparato burocrático profesional, neutral e independiente de la esfera política mediante la estabilidad burocrática ante los cambios de gobierno. El análisis del deterioro de los servicios civiles no consiste únicamente en analizar las grandes reformas administrativas o modificaciones legales, sino que también implica la observación de fenómenos "micro" que suceden en las interacciones entre los miembros pertenecientes al sistema y de las prácticas y estructuras informales que dan forma a la experiencia del patronazgo. Esta aproximación metodológica nos ayuda a entender cómo se han institucionalizado prácticas que son "invisibles" a la luz de las reglas formales. Así, un sistema de mérito puede verse sometido por el poder de los partidos políticos, debilitando, con ello, todas las funciones del Estado.

Este estudio de caso demostró que el patronazgo puede llegar a apoderarse de las estructuras formales, menoscabando todo el andamiaje que sustenta un sistema formal de gestión de recursos humanos mediante la manipulación y la simulación, aun en contextos apoyados por instituciones sólidas, como son las del gobierno federal estadounidense. El servicio civil en Puerto Rico es el ejemplo de una combinación de un sistema basado en el mérito profesional y otro basado en 
una dominación tradicional ejercida mediante prácticas neopatrimoniales. A pesar de contar con una sólida legislación que respalda al servicio civil, penalizando la discriminación por distintos motivos, en el sistema formal se presentan permanentemente casos de discriminación por razones políticas tras los continuos cambios en el gobierno.

Estos dos sistemas - el servicio civil y el patronazgo-, que pareciesen incompatibles por representar valores contrarios, han logrado adaptarse por la dominación política ejercida por los partidos sobre los empleados de carrera. La realidad de la administración pública en Puerto Rico muestra que los dos principales partidos políticos, cada vez que llega su oportunidad de gobernar, intentan, mediante prácticas informales institucionalizadas, construir capital político y financiero echando mano de las estructuras e instituciones formales del Estado.

El estudio de caso demuestra que en los sistemas de gestión de recursos humanos híbridos donde las prácticas de dominación informales son preponderantes, se termina por cooptar a las personas obligándolas a tomar una postura parcial en vez de conducirse neutralmente como la legislación del servicio civil exige. Asimismo, estas estructuras informales han consolidado prácticas donde los partidos políticos han logrado construir estructuras paralelas para generar ingresos financieros que servirán para seguir alimentando la maquinaria clientelar y electoral, haciendo que sea mucho más complejo y difícil terminar con estas prácticas ilegales e inconstitucionales.

Por otra parte, este estudio de caso reafirma que los remedios legales y las penalidades respaldadas por cortes estatales y federales no son suficientes para mantener un sistema de mérito o para disminuir las prácticas del patronazgo. El estudio de caso resalta algunas de las estructuras y de los mecanismos informales que influyen en que empleados de carrera sean administrados políticamente, y señala algunas de las lecciones que los empleados aprenden de su experiencia: aprenden que la neutralidad política no es un atributo deseable, aprenden a confiar en las personas que son del mismo partido y a desconfiar de las personas del partido de la oposición, aprenden a discriminar e internalizan la expectativa de que serán discriminados o favorecidos con base en su afiliación política. ¿Cuál será la mejor manera de frenar las actividades de estos grupos que activamente refuerzan estas prácticas, que movilizan la desconfianza? ¿Cómo se atiende la desconfianza? ¿Cómo se desaprende lo asimilado?

En este caso se presentó una discusión respecto al análisis y descubrimientos de entrevistas y revisión documental, tomando como referencias las reglas formales e informales del servicio civil en Puerto Rico. El análisis da luz a las interacciones 
que doblegan la voluntad y espíritu laboral de los empleados de carrera ante los designados políticos y otros actores políticos en las agencias. Esta dominación ejercida por la esfera de lo político permite que un sistema de servicio civil sirva como fachada a un sistema neopatrimonial de gestión de recursos humanos. Como expresó una empleada de carrera, en el gobierno de Puerto Rico: "no existe el mérito, no existe”. Gミิ

\section{REFERENCIAS}

Arellano Gault, D., A. Medina y R. Rodríguez (2018), "Servicio civil: Estabilidad y espíritu de cuerpo contra la corrupción”, en D. Arellano (ed.), ¿Podemos reducir la corrupción en México? Limites y posibilidades de los instrumentos a nuestro alcance, Ciudad de México, CIDE, pp. 177-205.

Arellano Gault, D. y M. Castillo Salas (eds.) (2019), La resbaladilla de la corrupción: Estudios sobre los procesos sociales y organizacionales de la corrupción colusiva en el sector público, Ciudad de México, CIDE.

Bearfield, D.A. (2009), "What Is Patronage? A Critical Reexamination", Public Administration Review, 69(1), pp. 64-76.

Box, R. (2015), Public Service Values, Nueva York, M.E. Sharpe.

Caballero-Fuentes, A. (2012), "Discrimen político en el sistema de recursos humanos del servicio público de Puerto Rico", presentado en la XXIX Conferencia y Exposición shrm, Fajardo, Puerto Rico, disponible en: http://shrmpr.shrm.org [fecha de consulta: 26 de septiembre de 2012].

Charron, N., C. Dahlström, M. Fazekas y V. Lapuente (2017), "Careers, Connections, and Corruption Risks: Investing the Impact of Bureaucratic Meritocracy on Public Procurement Processes", Journal of Politics, 79(1), pp. 89-104.

Chudnovsky, M. (2017), "La tensión entre mérito y confianza en la alta dirección pública de América Latina”, Revista del CLAD Reforma y Democracia, 69, pp. 5-40.

Chudnovsky, M. y M. Cafarelli (2018), "Los cambios en las estructuras organizacionales del Estado y su vínculo con la composición del empleo público: Argentina, 2003-2016”, Foro Internacional, 58(2), pp. 275-312.

Colón-González, J.L. (2012), "El discrimen político en el empleo público mediante la manipulación de los sistemas de mérito: El caso de Puerto Rico", presentado en el XVII Congreso Internacional del CLAD "Sobre la reforma del Estado y de la administración pública”, Cartagena, 30 de octubre- 2 de noviembre.

Comisión de Derechos Civiles (1993), Informe sobre discrimen politico en el empleo público en Puerto Rico, disponible en: https://cdc.pr.gov/InstitutoDeEducacion/RecursosEducativos/Pages/default.aspx [fecha de consulta: 22 de septiembre de 2021]. 
Comisión de Gobierno (2006), Informe Positivo P. de la C. 1359.

Cooper, C.A. (2020), "Politicization of the Bureaucracy across and within Administrative Traditions", International Journal of Public Administration, 44(7), pp. 1-14.

Cordero-Nieves, Y., A. Caballero-Fuentes, W. Vazquez-Irizarry y E.V. Segarra-Alméstica (2016), El discrimen político en el empleo público, San Juan, CEMGAP.

Cordero-Nieves, Y. (2012a), "El discrimen político en el empleo público”, en J.J. ColónMorera e I. Alegría-Ortega (eds.), Puerto Rico y los derechos humanos: Una intersección plural, San Juan, Ediciones Callejón.

Cordero-Nieves, Y. (2012b), "El estudio del discrimen político en el empleo público: Desafíos iniciales", presentado en el XVII Congreso Internacional del CLAD "Sobre la reforma del Estado y de la administración pública", Cartagena, 30 de octubre-2 de noviembre.

Cordero-Nieves, Y. (2014), "Consideraciones sobre los posibles efectos de la descentralización en la administración del servicio público: El caso de las regiones autónomas en Puerto Rico", Revista Estado, Gobierno y Gestión Pública, 24, pp. 5-33.

Dahlström, C., V. Lapuente y J. Teorell (2012), "Public Administration around the World", en S. Holmberg y B. Rothstein (eds.), Good Government: The Relevance of Political Science, Cheltenham, Edward Elgar, pp. 13-39.

Durazo, J. (2010), "Neo-Patrimonialism and Subnational Authoritarianism in Mexico: The Case of Oaxaca”, Journal of Politics in Latin America, 2(2), pp. 85-112.

Dussauge Laguna, M.I. (2011), “The Challenges of Implementing Merit-Based Personnel Policies in Latin America: Mexico's Civil Service Reform Experience”, Journal of Comparative Policy Analysis, 13(1), pp. 51-73.

Eisenstadt, S. (1973), Traditional Patrimonialism and Modern Neopatrominialism, Londres, Sage Publications.

Emerson, R., R. Fretz y L Shaw (1995), Writing Ethnographic Fieldnotes, Chicago, University of Chicago Press.

Erdmann, G. (2002), "Neo-Patrimonial Rule: Transition to Democracy has not Succeeded”, D+C Development and Cooperation, 29(1), pp. 9-11.

Erdmann, G. y U. Engel (2007), "Neopatrimonialism Reconsidered: Critical Review and Elaboration of an Elusive Concept”, Commonwealth \& Comparative Politics, 45(1), pp. 95-119.

Fuenzalida, J. y N. Riccucci (2019), “The Effects of Politicization on Performance: The Mediating Role of Hrm Practices", Review of Public Personnel Administration, 39(4), pp. 544-569.

Geddes, B. (1994), Politician's Dilemma: Building State Capacity in Latin America, Berkeley, University of California Press. 
Goodsell, C. (1965), Administration of a Revolution: Executive Reform In Puerto Rico Under Governor Tugwell, 1941-1946, Massachusetts, Harvard University Press.

Grindle, M.S. (2012), Jobs for the Boys: Patronage and the State in Comparative Perspective, Cambridge, Harvard University Press.

Hamilton, D.K. (2010), "Patronage in Illinois: The Political Subjugation of Public Administration", Review of Public Personnel Administration, 30(2), pp. 137-165.

Heidenheimer, A.J., M. Johnston y V.T. LeVine (1989), “The Persistence of Patronage Systems: An Introduction”, en A.J. Heidenheimer, M. Johnston y V.T. LeVine (eds.), Political Corruption: A Handbook, New Brunswick, Transaction Publishers, pp. 305-306. Iacoviello, M. y L. Zuvanic (2006), “Desarrollo e integración de la gestión de recursos humanos en los estados latinoamericanos", Documentos y Aportes en Administración Pública y Gestión Estatal, 6(7), pp. 45-92.

Iacoviello, M., M. Llano y C. Ramos (2017), "Alta dirección pública latinoamericana: Marchas y contramarchas", Revista de Gestión Pública, 6(2), pp. 173-214.

Ingraham, P.W. (1995), The Foundation of Merit: Public Service in American Democracy, Baltimore, Johns Hopkins University Press.

Instituto de Estadísticas de Puerto Rico (2021), Estadísticas de puestos ocupados en el gobierno, enero, disponible en: https://estadisticas.pr/en/inventario-de-estadisticas/puestos_ocupados_en_gobierno [fecha de consulta: 12 de marzo de 2021].

Ippolito-O’Donnell, G. (2006), "Political Clientelism and the Quality of Democracy”, presentado en el XX World Congress of the International Political Science AssociationIPSA, Fukuoka, julio, pp. 9-13.

Jancsics, D. (2019), “Corruption as Resource Transfer: An Interdisciplinary Synthesis”, Public Administration Review, 79(4), pp. 523-537.

Johnston, M. (1979), "Patrons and Clients, Jobs and Machines: A Case Study of the Uses of Patronage", American Political Science Review, 73(2), pp. 385-398.

Kearney, R.C. (1986), "Spoils in the Caribbean: The Struggle for Merit-Based Civil Service in the Dominican Republic", Public Administration Review, 46(2), pp. 144-151.

Klingner, D.E. (1996), "Public Personnel Management and Democratization: A View from Three Central American Republics”, Public Administration Review, 56(1), pp. 390-399.

Ley de Personal del Servicio Público de Puerto Rico (L.P.R.A.), disponible en: https:// bvirtualogp.pr.gov/ogp/Bvirtual/leyesreferencia/PDF/5-1975.pdf [fecha de consulta: 25 de febrero de 2021].

Light, P. (2006), “The Tides of Reform Revisited: Patterns in Making Government Work, 1945-2002”, Public Administration Review, 66(1), pp. 6-19.

Llorens, J.J., D. Klingner y J. Nalbandian (2017), Public Personnel Management: Context and Strategies, Nueva York, Routledge. 
Lomnitz, L.A. (1988), "Informal Exchange Networks in Formal Systems: A Theoretical Model”, American Anthropologist, 91(1), pp. 42-55.

Martínez Puón, R. (2006), "Alcances y resultados del servicio profesional de carrera en México: Un ejercicio de evaluación a tres años de su implementación”, Gestión y Politica Pública, XV(2), pp. 457-486.

Meyer-Sahling, J.H., K.S. Mikkelsen y C. Schuster (2018), "Civil Service Management and Corruption: What We Know and What We Don't", Public Administration, 96(2), pp. 276-285.

Meyer-Sahling, J.H. y K.S. Mikkelsen (2016), “Civil Service Laws, Merit, Politicization, and Corruption: The Perspective of Public Officials from Five East European Countries", Public Administration, 94(4), pp. 1105-1123.

Meza, O. y E. Pérez-Chiqués (2020), "Corruption Consolidation in Local Governments: A Grounded Analytical Framework”, Public Administration, Dor: https://doi.org/10.1111/ padm.12698.

Monsiváis A. y A. del Río (2013), "El neopatrimonialismo a debate: Coordenadas conceptuales y apuntes analíticos", Espiral, Estudios sobre Estado y Sociedad, 20(58), pp. 37-66.

Nieto, F. y M.C. Pardo (2019), "La implementación del servicio profesional de carrera, 2003-2012”, en G. Cejudo, M.C. Pardo y M. Dussauge (eds.), Variaciones de implementación: Ocho casos de política pública, Ciudad de México, CIDE.

Nigro, L.G., F.A. Nigro y E.J. Kellough (2007), "Public Employees - Rights and Responsibilities”, en L.G. Nigro, F.A. Nigro y E.J. Kellough (eds.), The New Public Personnel Administration, 6a. ed., Boston, Wadsworth, pp. 233-247.

O’Dwyer, C. (2006), Runaway State-building: Patronage Politics and Democratic Development, Baltimore, The Johns Hopkins University Press.

Oficina del Gobernador (1959), Informe del Comité del Gobernador para el Estudio de los Derechos Civiles en Puerto Rico, San Juan, Editorial del Colegio de Abogados.

Oliveros, V. (2013), "A Working Machine: Patronage Jobs and Political Services in Argentina", tesis doctoral, Columbia University,

Oliveros, V. (2016), "Making it personal: Clientelism, Favors, and the Personalization of Public Administration in Argentina", Comparative Politics, 48(3), pp. 373-391.

Oliveros, V. y C. Schuster (2018), "Merit, Tenure, and Bureaucratic Behavior: Evidence from a Conjoint Experiment in the Dominican Republic", Comparative Political Studies, 51(6), pp. 759-792.

Oszlak, O. (1986), "Public Policy and Political Regimes in Latin America", International Social Science Journal, 38(2), pp. 219-236.

Ozturk, M.B. (2005), "Corruption, Job Patronage, and the Political Economy of Human Capital Investment", tesis doctoral, University of Chicago, Department of Political Science. 
Pacheco-Muñiz, E. y H. Vera-Rodríguez (2009), "La administración de recursos humanos en el gobierno de Puerto Rico: Centralización, descentralización, control y caos", presentado en el XIV Congreso Internacional del CLAD "Sobre la reforma del Estado y de la administración pública”, Salvador de Bahía, 27-30 de octubre.

Pardo, M.C. (2005), "El servicio profesional de carrera en México: De la tradición al cambio", Foro Internacional, 45(4), pp. 599-634.

Peters, B.G. y J. Pierre (2004), "Politicization of the Civil Service: Concepts, Causes, Consequences", en B.G. Peters y J. Pierre (eds.), The Politicization of the Civil Service in Comparative Perspective, Nueva York, Routledge. pp. 13-25.

Pérez-Chiqués, E. y E. Rubin (2021), "Debasement of Merit: The Method and Experience of Political Discrimination by Public Employees in the Commonwealth of Puerto Rico", Review of Public Personnel Administration, Dor: https://doi.org/10.1177/ $0734371 X 211014948$.

Puentes, G. (2017), "Fricciones entre las ramas del poder público a propósito de la función pública en Colombia”, Estudios Socio-Jurídicos, 19(1), pp. 79-123.

Ramió, C. (2008), "La profesionalización de las agencias reguladoras en América Latina: Los casos de Perú y de República Dominicana”, en F. Longo y C. Ramió (eds.), La profesionalización del empleo público en América Latina, Barcelona, Fundación СıDOB, pp. 263-312.

Remmer, K.L. (1989), "Neopatrimonialism: The Politics of Military Rule in Chile, 19731987", Comparative Politics, 21(2), pp. 149-170.

Repucci, S. (2012), "Civil Service Reform: A Review”, documento de trabajo 2012/90, Helsinki, UNU-WIDER.

Roth, G. (1968), "Personal Rulership, Patrimonialism, and Empire-Building in the New States", World Politics, 20(2), pp. 194-206.

Ruiz-Acevedo, Á. (1996), "La glorificación y crucifixión del sistema de mérito en Puerto Rico”, Revista de Administración Pública, 29(1), pp. 13-28.

Santana, L. (1994), Fulgor y decadencia de la administración pública en Puerto Rico, San Juan, La Torre del Viejo.

Scott, J.C. (1969), "Corruption, Machine Politics, and Political Change", The American Political Science Review, 63(4), pp. 1142-1158.

Schuster, C. (2016), "What Causes Patronage Reform? It Depends on the Type of Civil Service Reform”, Public Administration, 94(4), pp. 1094-1104.

Schuster, C. (2017), "Legal Reform Need Not Come First: Merit-based Civil Service Management in Law and Practice", Public Administration, 95(3), pp. 571-588.

Senado de Puerto Rico (2016), Transcripción Vista Pública, Comisión Especial para el Estudio de las Normas y Procedimientos Relacionados con la Compra y Uso de Petró- 
leo por la Autoridad de Energía Eléctrica. Celebrada el martes, 2 de febrero de 2016, Presidida por el Hon. Aníbal J. Torres Torres, p. 117.

Stokes, S.C. (2005), "Perverse Accountability: A Formal Model of Machine Politics with Evidence from Argentina”, American Political Science Review, 99(3), pp. 315-325.

Van Riper, P.P. (1958), History of the United States Civil Service, Nueva York, Row, Peterson and Company.

Weber, Max (2002), Economía y Sociedad, Ciudad de México, Fondo de Cultura Económica. Wilson, W. (1981), “The Study of Administration”, en F.C. Mosher (ed.), Basic Literature of American Public Administration 1787-1950, Nueva York, Holmes \& Meier, pp. 66-81.

Elizabeth Pérez-Chiqués es doctora en Administración y Políticas Públicas, profesorainvestigadora adscrita a la División de Administración Pública del Centro de Investigación y Docencia Económicas (CIDE). Sus principales líneas de investigación están centradas en la corrupción gubernamental, en la gestión de los recursos humanos en el sector público y en la implementación de políticas públicas.

Edgar O. Bustos es doctor en Políticas Públicas por el Centro de Investigación y Docencia Económicas (CIDE). Sus principales líneas de investigación están centradas en la gestión de los recursos humanos en el sector público. 